\title{
Hemorheological alterations in adults with prediabetes identified by hemoglobin A1c levels
}

\author{
M.A. Marini ${ }^{\text {a,1 }}$, T.V. Fiorentino ${ }^{\text {b, }}{ }^{\text {, F. Andreozzi }}{ }^{\text {b }}$, G.C. Mannino ${ }^{\text {b }}$, E. Succurro ${ }^{\text {b }}$, \\ A. Sciacqua ${ }^{\text {b }}$, F. Perticone ${ }^{b}$, G. Sesti $^{\text {b,* }}$ \\ ${ }^{a}$ Department of Systems Medicine, University of Rome Tor Vergata, 00133 Rome, Italy \\ ${ }^{b}$ Department of Medical and Surgical Sciences, University Magna-Græcia of Catanzaro, Viale Europa, 88100 Catanzaro, Italy \\ Received 24 January 2017; received in revised form 11 April 2017; accepted 11 April 2017 \\ Available online
}

\section{KEYWORDS \\ Blood viscosity; \\ Hematocrit; \\ Prediabetes; \\ HbA1c; \\ Cardiovascular risk}

\begin{abstract}
Background and aims: A link between increased blood viscosity and type 2 diabetes has been previously reported. Herein, we investigated the association of blood viscosity with prediabetes, identified by glycated hemoglobin A1c (HbA1c) according to the new American Diabetes Association criteria, and subclinical atherosclerosis.

Methods and results: The study cohort includes 1136 non-diabetic adults submitted to anthropometrical evaluation, an oral glucose tolerance test and ultrasound measurement of carotid intima-media thickness (IMT). Whole blood viscosity was estimated using a validated formula based on hematocrit and total plasma proteins.

After adjusting for age, and gender, individuals with HbA1c-defined prediabetes (HbA1c 5.7-6.4\% [39-47 mmol/mol]) exhibited significantly higher values of hematocrit, and predicted blood viscosity as compared with controls. Increased levels of IMT were observed in subjects with HbA1c-defined prediabetes in comparison to controls. Predicted blood viscosity was positively correlated with age, waist circumference, blood pressure, cholesterol, triglycerides, fibrinogen, white blood cell, HbA1c, fasting and 2-h post-load glucose levels, fasting insulin, IMT and inversely correlated with HDL and Matsuda index of insulin sensitivity. Of the three glycemic parameters, i.e. HbA1c, fasting and 2h post-load glucose, only HbA1c showed a significant correlation with predicted blood viscosity $(\beta=0.054, P=0.04)$ in a multivariate regression analysis model including multiple atherosclerosis risk factors.

Conclusion: The study shows that individuals with HbA1c-defined prediabetes have increased predicted blood viscosity and IMT. The HbA1c criterion may be helpful to capture individuals with an increased risk of diabetes and cardiovascular disease who may benefit from an intensive lifestyle intervention.

๑ 2017 The Italian Society of Diabetology, the Italian Society for the Study of Atherosclerosis, the Italian Society of Human Nutrition, and the Department of Clinical Medicine and Surgery, Federico II University. Published by Elsevier B.V. All rights reserved.
\end{abstract}

\footnotetext{
* Corresponding author. Fax: +390961 3647192.

E-mail address: sesti@unicz.it (G. Sesti).

${ }^{1}$ These authors equally contributed to the work.
}

\section{Introduction}

The term prediabetes refers to dysglycemic conditions at high-risk for developing type 2 diabetes and cardiovascular disease [1]. The most commonly studied categories of prediabetes are impaired fasting glycemia (IFG) and impaired glucose tolerance (IGT). Individuals with IFG or IGT have shown a high conversion rate to type 2 diabetes 
and significant efforts have been devoted to the identification of lifestyle or pharmacological intervention able to prevent or delay onset of type 2 diabetes and its complications in these high-risk categories [2]. Several prospective studies that used glycated hemoglobin A1c (HbA1c) levels to predict the progression to type 2 diabetes have shown a strong, continuous association between HbA1c values and subsequent overt diabetes [3,4]. In 2010, the American Diabetes Association (ADA) has introduced HbA1c as a diagnostic test option for type 2 diabetes and prediabetes in addition to fasting and 2$\mathrm{h}$ plasma glucose levels during an oral glucose tolerance test (OGTT) [1]. According to the ADA recommendations [1], a HbA1c value of $5.7-6.4 \%(39-47 \mathrm{mmol} / \mathrm{mol})$ identifies individuals with prediabetes. HbA1c is an integrated measure of mean glycemia and offers some practical advantages over measurements of fasting plasma glucose or glucose levels during an OGTT including not requiring fasting, having greater pre-analytical stability, and showing lower between and within-subject variations as well as less day-to-day perturbations during periods of stress and illness. Notably, the three metabolic parameters used as measures of prediabetes, i.e. fasting plasma glucose, 2-h post-load glucose, and HbA1c, reflect different aspects of glucose homeostasis with fasting plasma glucose being a marker of steady-state glucose metabolism, 2-h post-load glucose being an indicator of response to glycemic stress, and HbA1c being a marker of average blood glucose levels over the previous 2-3 months. As a consequence, it is not surprising that poor concordance between glucose tolerance groups identified by fasting plasma glucose, 2-h post-load glucose, and HbA1c has been reported in different ethnic groups [5-7].

There is increasing evidence suggesting that increased blood viscosity, i.e. the resistance to flow in a blood vessel, is associated with cardio-vascular risk factors including dyslipidemia, hyperglycemia, hypertension, smoking, obesity, and metabolic syndrome [8-12]. Blood viscosity is inversely correlated to flow and raised blood viscosity might therefore play a role in determining insulin resistance and type 2 diabetes by limiting delivery of glucose, and insulin to target tissues such as the skeletal muscle [13]. Cross-sectional studies have shown significant independent association of hematocrit, the most important parameter of blood viscosity, or blood viscosity itself with IFG condition, and prospective studies have shown their associations with incident type 2 diabetes [12,14,15]. These data suggest that an increase in blood viscosity may contribute to the development of type 2 diabetes in individuals at high-risk such those having dysglycemic conditions. However, whether HbA1c-defined prediabetes is associated with elevated blood viscosity is still unsettled. In consideration of the expected increase in the use of HbA1c as a screening tool to identify individuals with dysglycemic conditions, in the present study, we tested the hypothesis that elevated estimated whole blood viscosity would be cross-sectionally associated with HbA1cdefined prediabetes and higher levels of carotid intima- media thickness (IMT), an indicator of subclinical vascular damage [16], in a large cohort of wellcharacterized nondiabetic adults.

\section{Methods}

\section{Study subjects}

The study cohort included 1136 White individuals consecutively recruited at the Department of Systems Medicine of the University of Rome-Tor Vergata and at the Department of Medical and Surgical Sciences of the University "Magna Graecia" of Catanzaro, as previously described $[6,7,17]$. On the first day, after 12-h fasting, subjects underwent anthropometrical evaluation, including body mass index (BMI), and waist circumference, and a venous blood sample was drawn for laboratory determinations. Blood pressure was measured in the sitting position, after five minutes of quiet rest. On the second day, after a 12-h fasting, an OGTT was performed with sampling for plasma glucose and insulin.

High resolution B-mode ultrasound was used to measure IMT of the common carotid artery using an ATL HDI 3000 ultrasound system (Advanced Technology Laboratories, Bothell, WA) equipped with a $7.5 \mathrm{MHz}$ transducer, as previously described [6].

The study was approved by the local ethics committee (Comitato Etico Azienda Ospedaliera "Mater Domini"). Written informed consent was obtained from each subject in accordance with principles of the Declaration of Helsinki.

\section{Analytical determinations}

Hemoglobin, hematocrit, and white blood cell count were determined using an automated particle counter (Siemens Healthcare Diagnostics ADVIA ${ }^{\circledR}$ 120/2120 Haematology System, Milan, Italy). Glucose, triglycerides, total and high density lipoprotein (HDL) cholesterol concentrations were determined by enzymatic methods (Roche, Basel, Switzerland). HbA1c was measured with high performance liquid chromatography using a National Glycohemoglobin Standardization Program (NGSP) certified automated analyzer (Adams HA-8160 HbA1C analyzer, Menarini, Italy). Fibrinogen was measured by an automated nephelometric technology using the BNTMII System analyzer (Siemens Healthcare, Italy). Plasma insulin concentration was measured with a chemiluminescencebased assay (Immulite ${ }^{\circledR}$, Siemens Healthcare $\mathrm{GmbH}$, Erlangen, Germany).

\section{Calculations}

Whole blood viscosity at $208 \mathrm{~s}^{-1}$ of shear rate was estimated by a previously validated formula that takes into account hematocrit and plasma proteins $[15,18,19]$ : Predicted whole blood viscosity $=\left[\begin{array}{lll}0.12 & \times\end{array}\right]+$ [0.17 $\times(p-2.07)]$, where $h$ is hematocrit (\%) and $p$ is plasma protein concentration $(\mathrm{g} / \mathrm{dl})$. 
The Matsuda index of insulin sensitivity was calculated as follows: 10.000/square root of [FPG $\times$ fasting insulin] $\times$ [mean glucose $\times$ mean insulin during OGTT] [20] .

Individuals were classified as having normal glucose tolerance (NGT) when fasting plasma glucose was $<100 \mathrm{mg} / \mathrm{dl}$ and 2 -h post-load plasma glucose was $<140 \mathrm{mg} / \mathrm{dl}$, or IGT when fasting plasma glucose was $<126 \mathrm{mg} / \mathrm{dl}$ and 2 -h post-load plasma glucose was $140-199 \mathrm{mg} / \mathrm{dl}[1]$.

\section{Statistical analysis}

Variables with skewed distribution including triglycerides, fasting, and 2-h insulin were natural log transformed for statistical analyses. Continuous data are expressed as mean \pm SD. Categorical variables were compared by $\chi^{2}$ test. We used a general linear model with adjustment for age and gender to test pairwise differences in anthropometric, metabolic, and hematological parameters among HbA1c-defined prediabetes groups. Pearson correlation coefficient was used to test the correlation between continuous variables. Relationships between variables were sought by a multiple linear regression analysis in order to assess their independent contribution to hematological parameters. The variance inflection factor (VIF) was less than 2 in all the analyses indicating that multicollinearity among variables was not a problem in the multiple regression model. Two-sided $P$ value $<0.05$ was considered statistically significant. All analyses were performed using the statistical package SPSS 22.0 for Windows (SPSS, $\mathrm{IBM}^{\circledR}$, Chicago, IL).

\section{Results}

\section{Cardio-metabolic characteristics}

The 1136 participants to the study were stratified into two groups on the basis of their HbA1c levels, according to the ADA recommendation [1]. Of the individuals who were classified as at increased risk of type 2 diabetes based on HbA1c levels, 186 (50.8\%) had NGT, and 180 (49.2\%), had IGT according to the OGTT results. Poor agreement existed between HbA1c and 2-h PG criteria for identification of individuals with pre-diabetes $(\kappa$ coefficient $=0.119$ ), indeed only $15.8 \%$ of individuals had prediabetes based on both HbA1c and OGTT criteria.

Anthropometric and metabolic characteristics of the study cohort, according to HbA1c-defined prediabetes classification are reported in Table 1. Gender, and age distributions were unevenly scattered among the two groups: subjects with HbA1c-defined prediabetes were older and more likely to be men than women (Table 1 ). Since, these two parameters are associated with hemorheological features, all subsequent analyses were adjusted for age, and gender. No differences between the two groups were observed with respect to smoking state.

By design, individuals with HbA1c-defined prediabetes exhibited higher levels of fasting and 2-h post-load glucose (Table 1). Individuals with HbA1c-defined prediabetes had a worse metabolic risk profile as compared to the control group. After adjusting for age, and gender, individuals with HbA1c-defined prediabetes exhibited significantly higher values of BMI, waist circumference, triglycerides, fibrinogen, fasting insulin levels, and lower

Table 1 Anthropometric and metabolic characteristics of the study subjects stratified according to HbA1c levels.

\begin{tabular}{|c|c|c|c|}
\hline Variables & $\begin{array}{l}\mathrm{HbA} 1 \mathrm{c}<5.7 \% \\
(39 \mathrm{mmol} / \mathrm{mol})\end{array}$ & $\begin{array}{l}\mathrm{HbA} 1 \mathrm{c} 5.7-6.4 \% \\
(39-47 \mathrm{mmol} / \mathrm{mol})\end{array}$ & $P$ \\
\hline Number (male/female) & $770(313 / 457)$ & $366(211 / 155)$ & $<0.0001$ \\
\hline Age $(y r)$ & $43 \pm 13$ & $51 \pm 12$ & $<0.0001^{*}$ \\
\hline Current smokers (\%) & 167 (21.7\%) & $83(22.7 \%)$ & 0.70 \\
\hline BMI $\left(\mathrm{kg} / \mathrm{m}^{2}\right)$ & $29.3 \pm 6.7$ & $31.9 \pm 6.8$ & $<0.0001$ \\
\hline Waist circumference $(\mathrm{cm})$ & $99.0 \pm 15.5$ & $107.0 \pm 16.1$ & $<0.0001$ \\
\hline Systolic blood pressure (mmHg) & $124 \pm 17$ & $128 \pm 16$ & 0.95 \\
\hline Diastolic blood pressure (mmHg) & $77 \pm 11$ & $79 \pm 11$ & 0.37 \\
\hline Total cholesterol (mg/dl) & $194 \pm 37$ & $196 \pm 38$ & 0.45 \\
\hline HDL cholesterol (mg/dl) & $52 \pm 14$ & $48 \pm 12$ & $<0.0001$ \\
\hline Triglycerides (mg/dl) & $114 \pm 69$ & $141 \pm 74$ & $<0.0001$ \\
\hline Fibrinogen (mg/dl) & $300 \pm 75$ & $311 \pm 73$ & 0.04 \\
\hline $\mathrm{HbA1c}(\%)[\mathrm{mmol} / \mathrm{mol}]$ & $5.3 \pm 0.2[34 \mathrm{mmol} / \mathrm{mol}]$ & $5.9 \pm 0.2[41 \mathrm{mmol} / \mathrm{mol}]$ & $<0.0001$ \\
\hline IGT (\%) & $159(20.6 \%)$ & $180(49.2 \%)$ & $<0.0001$ \\
\hline Fasting glucose (mg/dl) & $89 \pm 10$ & $98 \pm 11$ & $<0.0001$ \\
\hline 2-h glucose (mg/dl) & $115 \pm 29$ & $134 \pm 30$ & $<0.0001$ \\
\hline Fasting insulin $(\mu \mathrm{U} / \mathrm{ml})$ & $12 \pm 8$ & $17 \pm 9$ & $<0.0001$ \\
\hline $\begin{array}{l}\text { Matsuda insulin sensitivity index } \\
\left(\mathrm{mg} \mathrm{L}^{2} \mathrm{mmol}^{-1} \mathrm{mU}^{-1} \mathrm{~min}^{-1}\right)\end{array}$ & $79 \pm 51$ & $52 \pm 33$ & $<0.0001$ \\
\hline Intima-media thickness (mm) & $0.69 \pm 0.18$ & $0.75 \pm 0.16$ & $<0.0001$ \\
\hline
\end{tabular}

Data are means \pm SD. Triglycerides, fasting and 2-h insulin were log transformed for statistical analysis, but values in the table represent back transformation to the original scale. Comparisons between the two groups were performed using a general linear model with adjustment for age and gender. $P$ values refer to results after analyses with adjustment for age, and gender. ${ }^{*} P$ values refer to results after analyses with adjustment for gender. Categorical variables were compared by $\chi^{2}$ test. BMI: body mass index; HDL: high-density lipoprotein; IGT: impaired glucose tolerance. 
values of HDL cholesterol and Matsuda index of insulin sensitivity as compared with the control group (Table 1). Moreover individuals with HbA1c-defined prediabetes had higher levels of IMT in comparison to the control group (Table 1).

\section{Hemorheological characteristics}

Hemorheological features of the two study groups are presented in Table 2. After adjusting for age, and gender, individuals with $\mathrm{HbA1c}$-defined prediabetes exhibited significantly higher values of white blood cell count, hematocrit and predicted whole blood viscosity as compared to the control group (Table 2 and Fig. 1A,B); while no significant differences were observed with respect to total protein and hemoglobin concentrations.

Univariate correlations between predicted blood viscosity, hematocrit, total protein levels and anthropometric and metabolic variables in the whole study group are presented in Table 3. Predicted whole blood viscosity was significantly and positively correlated with age, waist circumference, systolic and diastolic blood pressure, cholesterol, triglycerides, fibrinogen, white blood cell count, HbA1c, fasting and 2-h post-load plasma glucose levels, fasting insulin, and inversely correlated with HDL cholesterol and Matsuda index of insulin sensitivity (Table 3). Similar results were observed for hematocrit.
Importantly, predicted blood viscosity and hematocrit were positively associated with IMT. Total protein concentrations were positively correlated with systolic and diastolic blood pressure, total cholesterol, triglycerides, fibrinogen, white blood cell count, fasting insulin, and negatively associated with age.

To estimate the independent contribution of variables to predicted whole blood viscosity, hematocrit and total protein levels, we carried out a multivariable regression analysis in a model including all the above variables significantly correlated with these hemorheological parameters (Table 4). Comparison of standardized coefficients allowed the determination of the relative strength of each trait association with predicted whole blood viscosity (listed from strongest to weakest): white blood cell count ( $\beta=0.145$, $P<0.0001)$, HDL cholesterol $(\beta=-0.126, P<0.0001)$, triglycerides $(\beta=0.109, P=0.001)$, total cholesterol $(\beta=0.104, P<0.0001)$, diastolic blood pressure $(\beta=0.087$, $P=0.005)$, age $(\beta=0.066, P=0.007)$, fibrinogen $(\beta=0.056, P=0.02)$, and HbA1c $(\beta=0.054, P=0.04)$ (Table 4 ). These factors explained $41.1 \%$ of the variance of predicted whole blood viscosity.

We found that the independent contributors of hematocrit were (listed from strongest to weakest): white blood cell count $(\beta=0.15, P<0.0001)$, diastolic blood pressure $(\beta=0.13, P=0.01)$, insulin sensitivity assessed by Matsuda Insulin Sensitivity index $(\beta=-0.12, P=0.05)$, age $(\beta=0.11$,

Table 2 Hemorheological characteristics of the study subjects stratified according to HbA1c levels.

\begin{tabular}{llll}
\hline Variables & $\begin{array}{l}\text { HbA1c }<5.7 \% \\
(39 \mathrm{mmol} / \mathrm{mol})\end{array}$ & $\begin{array}{l}\text { HbA1c 5.7-6.4\% } \\
(39-47 \mathrm{mmol} / \mathrm{mol})\end{array}$ & $P$ \\
\hline White blood cell count $\left(\times 10^{9} / \mathrm{l}\right)$ & $6715 \pm 1910$ & $7120 \pm 1862$ & $<0.0001$ \\
Hematocrit $(\%)$ & $41.6 \pm 4.1$ & $43.1 \pm 4.6$ & 0.04 \\
Hemoglobin $(\mathrm{g} / \mathrm{dl})$ & $13.7 \pm 1.4$ & $14.1 \pm 1.5$ & 0.61 \\
Total protein $(\mathrm{g} / \mathrm{l})$ & $7.16 \pm 0.45$ & $7.18 \pm 0.46$ & 0.51 \\
Predicted whole blood viscosity $(\mathrm{cP})$ & $5.80 \pm 0.51$ & $6.05 \pm 0.57$ & 0.04 \\
\hline
\end{tabular}

Data are means \pm SD. Comparisons between the study groups were performed using a general linear model with adjustment for age and gender. $P$ values refer to results after analyses with adjustment for age, and gender.

A

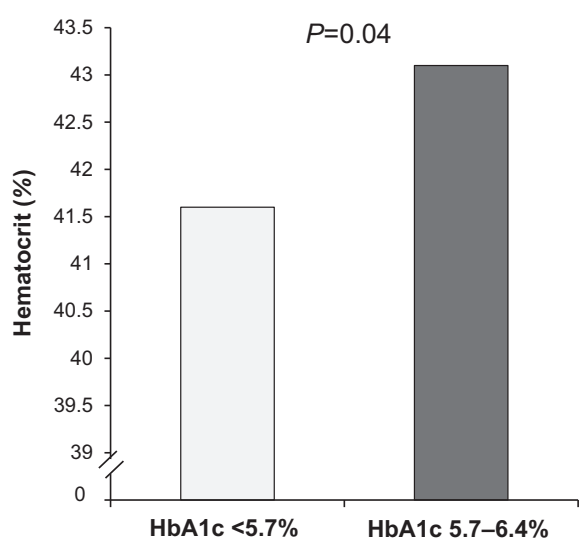

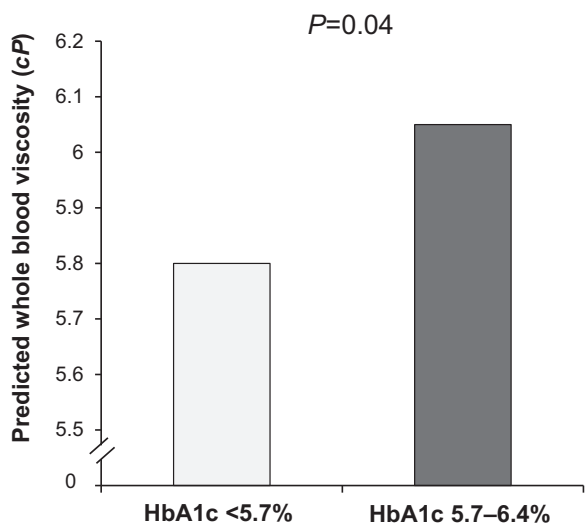

Figure 1 Hematocrit levels (A) and predicted whole blood viscosity (B) in study participants stratified according to HbA1c levels. 
Table 3 Univariate correlations between hemorheological parameters and anthropometric and metabolic variables.

\begin{tabular}{|c|c|c|c|c|c|c|}
\hline & \multicolumn{2}{|c|}{ Predicted whole blood viscosity } & \multicolumn{2}{|c|}{ Hematocrit } & \multicolumn{2}{|c|}{ Total protein } \\
\hline & $r$ & $P$ & $r$ & $P$ & $r$ & $P$ \\
\hline Age (years) & 0.18 & $<0.0001$ & 0.19 & $<0.0001$ & -0.05 & 0.05 \\
\hline $\operatorname{BMI}\left(\mathrm{kg} / \mathrm{m}^{2}\right)$ & 0.03 & 0.32 & 0.26 & 0.38 & 0.03 & 0.32 \\
\hline Waist circumference $(\mathrm{cm})$ & 0.16 & $<0.0001$ & 0.16 & $<0.0001$ & 0.02 & 0.57 \\
\hline Systolic blood pressure (mmHg) & 0.29 & $<0.0001$ & 0.28 & $<0.0001$ & 0.14 & $<0.0001$ \\
\hline Diastolic blood pressure (mmHg) & 0.28 & $<0.0001$ & 0.27 & $<0.0001$ & 0.14 & $<0.0001$ \\
\hline Total cholesterol (mg/dl) & 0.17 & $<0.0001$ & 0.16 & $<0.0001$ & 0.14 & $<0.0001$ \\
\hline HDL cholesterol (mg/dl) & -0.18 & $<0.0001$ & -0.18 & $<0.0001$ & -0.004 & 0.89 \\
\hline Triglycerides (mg/dl) & 0.28 & $<0.0001$ & 0.27 & $<0.0001$ & 0.17 & $<0.0001$ \\
\hline Fibrinogen (mg/dl) & 0.14 & $<0.0001$ & -0.022 & 0.47 & 0.20 & $<0.0001$ \\
\hline White blood cell count $\left(\times 10^{9} / 1\right)$ & 0.16 & $<0.0001$ & 0.15 & $<0.0001$ & 0.08 & 0.01 \\
\hline HbA1c (\%) [mmol/mol] & 0.10 & 0.001 & 0.12 & $<0.0001$ & 0.01 & 0.72 \\
\hline Fasting glucose (mg/dl) & 0.17 & $<0.0001$ & 0.18 & $<0.0001$ & -0.04 & 0.23 \\
\hline 2-h glucose $(\mathrm{mg} / \mathrm{dl})$ & 0.13 & $<0.0001$ & 0.13 & $<0.0001$ & 0.02 & 0.57 \\
\hline Fasting insulin $(\mu \mathrm{U} / \mathrm{ml})$ & 0.12 & $<0.0001$ & 0.11 & $<0.0001$ & 0.07 & 0.02 \\
\hline $\begin{array}{l}\text { Matsuda insulin sensitivity index } \\
\left(\mathrm{mg} \mathrm{L}^{2} \mathrm{mmol}^{-1} \mathrm{mU}^{-1} \mathrm{~min}^{-1}\right)\end{array}$ & -0.20 & $<0.0001$ & -0.20 & $<0.0001$ & -0.05 & 0.15 \\
\hline Intima-media thickness (mm) & 0.17 & $<0.0001$ & 0.16 & $<0.0001$ & 0.06 & 0.08 \\
\hline
\end{tabular}

BMI: body mass index; HDL: high-density lipoprotein.

Table 4 Multiple regression analysis with predicted whole blood viscosity, hematocrit or total protein as the dependent variable.

\begin{tabular}{|c|c|c|c|c|c|c|}
\hline & \multicolumn{2}{|c|}{ Predicted whole blood viscosity } & \multicolumn{2}{|l|}{ Hematocrit } & \multicolumn{2}{|l|}{ Total protein } \\
\hline & $\beta( \pm \mathrm{SE})$ & $P$ & $\beta( \pm \mathrm{SE})$ & $P$ & $\beta( \pm \mathrm{SE})$ & $P$ \\
\hline Age (years) & $0.066( \pm 0.001)$ & 0.02 & $0.11( \pm 0.01)$ & 0.03 & $-0.16( \pm 0.001)$ & $<0.0001$ \\
\hline Waist circumference $(\mathrm{cm})$ & $0.041( \pm 0.001)$ & 0.14 & $0.031( \pm 0.001)$ & 0.58 & $-0.04( \pm 0.001)$ & 0.42 \\
\hline Systolic blood pressure $(\mathrm{mmHg})$ & $0.032( \pm 0.001)$ & 0.32 & $0.076( \pm 0.01)$ & 0.08 & $0.13( \pm 0.001)$ & 0.01 \\
\hline Diastolic blood pressure $(\mathrm{mmHg})$ & $0.087( \pm 0.002)$ & 0.005 & $0.13( \pm 0.01)$ & 0.01 & $0.06( \pm 0.002)$ & 0.20 \\
\hline Total cholesterol (mg/dl) & $0.104( \pm 0.001)$ & $<0.0001$ & $0.09( \pm 0.001)$ & 0.02 & $0.078( \pm 0.001)$ & 0.05 \\
\hline HDL cholesterol (mg/dl) & $-0.126( \pm 0.001)$ & $<0.0001$ & $-0.09( \pm 0.01)$ & 0.03 & $0.08( \pm 0.001)$ & 0.05 \\
\hline Triglycerides (mg/dl) & $0.109( \pm 0.035)$ & 0.001 & $0.075( \pm 0.07)$ & 0.15 & $0.19( \pm 0.042)$ & $<0.0001$ \\
\hline Fibrinogen (mg/dl) & $0.056( \pm 0.001)$ & 0.02 & $-0.09( \pm 0.001)$ & 0.02 & $0.22( \pm 0.001)$ & $<0.0001$ \\
\hline White blood cell count $\left(\times 10^{9} / 1\right)$ & $0.145( \pm 0.001)$ & $<0.0001$ & $0.15( \pm 0.001)$ & $<0.0001$ & $-0.004( \pm 0.001)$ & 0.91 \\
\hline HbA1c (\%) [mmol/mol] & $0.054( \pm 0.001)$ & 0.04 & $0.08( \pm 0.02)$ & 0.05 & $-0.028( \pm 0.01)$ & 0.48 \\
\hline Fasting glucose (mg/dl) & $0.018( \pm 0.001)$ & 0.55 & $0.077( \pm 0.01)$ & 0.06 & $-0.005( \pm 0.002)$ & 0.89 \\
\hline 2 -h glucose $(\mathrm{mg} / \mathrm{dl})$ & $0.007( \pm 0.001)$ & 0.80 & $-0.026( \pm 0.01)$ & 0.56 & $0.04( \pm 0.001)$ & 0.33 \\
\hline Fasting insulin $(\mu \mathrm{U} / \mathrm{ml})$ & $0.059( \pm 0.035)$ & 0.10 & $-0.099( \pm 0.04)$ & 0.09 & $0.11( \pm 0.04)$ & 0.08 \\
\hline $\begin{array}{l}\text { Matsuda insulin sensitivity index } \\
\left(\mathrm{mg} \mathrm{L}^{2} \mathrm{mmol}^{-1} \mathrm{mU}^{-1} \mathrm{~min}^{-1}\right)\end{array}$ & $-0.016( \pm 0.001)$ & 0.67 & $-0.12( \pm 0.001)$ & 0.05 & $0.12( \pm 0.01)$ & 0.06 \\
\hline
\end{tabular}

$P=0.03)$, total cholesterol $(\beta=0.09, P=0.02)$, HDL cholesterol $(\beta=-0.09, P=0.03)$, fibrinogen $(\beta=0.09$, $P=0.02)$ and $\operatorname{HbA1c}(\beta=0.08, P=0.05)$. The full model explained $42 \%$ of the variance of hematocrit.

Furthermore, clinical variables independently associated with total protein concentrations were (listed from strongest to weakest): fibrinogen $(\beta=0.22, P<0.0001)$, triglycerides $(\beta=0.19, P<0.0001)$, age $(\beta=-0.16$, $P<0.0001)$; systolic blood pressure $(\beta=0.13, P=0.01)$, HDL cholesterol $(\beta=0.08, P=0.05)$ and total cholesterol ( $\beta=0.078, P=0.05$ ). The full model explained $34 \%$ of the variance of total protein levels.

\section{Discussion}

Measurement of $\mathrm{HbA} 1 \mathrm{c}$ has been recently recommended by ADA as a diagnostic test not only for diabetes but also for conditions at increased risk to develop diabetes
[1,3-5]. Both type 2 diabetes and HbA1c-defined prediabetes conditions are associated with an increased risk of cardiovascular disease [1,3,6,21-23] and an association between a higher whole blood viscosity, which is considered a cardiovascular risk factor $[8,18,19]$, and type 2 diabetes has been described $[14,15]$.

In this paper we report, for the first time, a significant association of predicted whole blood viscosity with a HbA1c value of $5.7-6.4 \%(39-47 \mathrm{mmol} / \mathrm{mol})$, a condition that confers increased risk to develop type 2 diabetes [1,4]. The findings of the present study extend the findings in people at risk for type 2 diabetes in several important ways. First, we assessed the association of hemorheological parameters with HbA1c in a large sample (1136 individuals) of nondiabetic subjects using a validated measure of whole blood viscosity $[15,18,19]$. The associations of hemorheological parameters with HbA1c-defined prediabetes in the present study were consistent, and a 
significant association of HbA1c with predicted whole blood viscosity and hematocrit was observed even after adjustment for multiple covariates. Notably, when we compare three glucose homeostasis parameters, i.e., HbA1c, fasting glucose, and 2-h post-load glucose levels, we observed that HbA1c was the only measure of glucose homeostasis that remained associated with predicted whole blood viscosity, and hematocrit in a multivariate regression analysis model including multiple atherosclerosis risk factors.

Importantly the hemorheological alterations detected in individuals with HbA1c-defined prediabetes were associated with subclinical vascular damage, as demonstrated by higher values of IMT, a known indicator of early atherosclerosis that predicts cardiovascular events [16].

The mechanism by which higher HbA1c levels are associated with elevated whole blood viscosity is undefined. Keeping in mind that several studies have reported that insulin resistance is associated with increased blood viscosity and erythrocyte aggregability [24,25], a greater degree of insulin resistance in these subjects is one possible explanation since previous studies employing the hyperinsulinemic euglycemic clamp technique to directly evaluate insulin sensitivity had shown that individual with HbA1c-defined prediabetes have impaired insulinstimulated glucose disposal [21,26]. Accordingly, as compared with control individuals, we observed that subjects with HbA1c-defined prediabetes have lower insulin sensitivity as assessed by a validated OGTT-derived index proxy of muscle insulin sensitivity [21]. A number of mechanisms have been proposed to explain the association of hematocrit, the primary determinant of whole blood viscosity or viscosity itself with insulin resistance. In normal conditions moderate increases in hematocrit and blood viscosity are associated to vasodilation induced by shear stress-mediated production of nitric oxide by endothelial cells. However, this compensatory response is reduced in conditions associated with endothelial dysfunction including impaired glucose homeostasis and insulin resistance [27]. In this regard it should be noted that we observed increased levels of IMT, a wellrecognized marker of early atherosclerosis [16], in individuals with $\mathrm{HbA1c}$-defined prediabetes. In presence of a reduced nitric oxide bioavailability, raised blood viscosity is associated with decreased flow. A reduction in blood flow, in turn, may lead to a decreased delivery of glucose to skeletal muscle [13] with consequent elevation in circulating glucose and insulin levels. On the other hand, hyperinsulinemia due to insulin resistance may cause vasoconstriction via sympathetic neural activation, which in turn would lead to hemoconcentration and increased blood viscosity [13]. Alternatively, compensatory hyperinsulinemia may decrease membrane fluidity of erythrocytes causing a disturbance in the blood rheological behavior and the microcirculation [28].

Additionally, chronic sub-clinical inflammation could be a common mechanistic factor since it has been associated with both blood viscosity [18], and HbA1c-defined prediabetes [29]. In the present study, we found that individuals with HbA1c-defined prediabetes have increased fibrinogen levels as compared with control subjects. An increase in fibrinogen may be an indicator of systemic inflammation but may also have a role in enhancing blood viscosity.

Increased lipoproteins levels may be another shared mechanism linking blood viscosity and HbA1c-defined prediabetes. Low HDL levels have been associated with an elevated blood viscosity $[9,18]$. Herein, we found that HbA1c-defined prediabetes exhibit a reduction in HDL levels as compared with control individuals, which was inversely related to whole blood viscosity. It has been suggested that HDL may compete with LDL binding to erythrocytes and antagonize LDL-induced erythrocyte aggregates, thus leading to a decrease in blood viscosity [9].

Finally, it is possible that chronic hyperglycemia captured by HbA1c measure can lead to hyperaggregation and low deformability of erythrocytes by changing hemoglobin and membrane proteins, as well as serum proteins including fibrinogen and globulins.

Strengths of the current study includes the large sample size, the inclusion of men and women, the demographically homogeneous group of adults from the same Caucasian ethnicity, wealth of detailed clinical, anthropometric and laboratory variables collected by medical professionals with a standardized protocol that allowed us to adjust for multiple confounders, the centralization of biochemical and hormonal analyses, the exclusion of confounding chronic disorders potentially affecting both hemoglobin concentration and inflammatory biomarkers, the exclusion of subjects treated with corticosteroids, antiplatelet, anticoagulant, lipid-lowering and hypoglycemic agents.

Nonetheless, our study has potential limitations that deserve mention. First, we had no direct measurement of blood viscosity. However, our estimates of whole blood viscosity were based on a prediction equation that has been validated in previous studies $[15,18,19]$. Secondly, we did not measure other blood components of blood viscosity, such as erythrocyte deformability and aggregability. It should be noticed that our large sample counterbalances the reduced precision of blood viscosity measures, and the strength and consistency of the observed associations using routine hematological measures may have helpful implications for clinical practice. In addition, participants underwent a single $75 \mathrm{~g}$ OGTT to determine glucose tolerance, and therefore, it is possible that some individuals might have been classified inappropriately. Moreover, all variables, including hematocrit, hemoglobin and total protein concentrations were measured once. Although such an approach is common in large observational studies, intra-individual variation in biochemical variables cannot be taken into account. Furthermore, the present study is based on opportunistic screening for recruitment of individuals considered at risk of type 2 diabetes and cardiovascular disease referred to a university hospital for further assessment. Additionally, all participants were Whites, and results might vary as a function of ethnic group. Indeed, there is evidence that 
HbA1c levels are higher among Blacks, Hispanics, American Indians, and Asian Americans compared to Whites even after adjustment for factors that might affect glycemia [30]. Finally, the cross-sectional design of the study reflects only an association of HbA1c with whole blood viscosity, and precludes us from discerning cause and effect using this design, but it provides some pathophysiological clues to justify the use of HbA1c as diagnostic criterion to identify individuals at risk of type 2 diabetes.

The present findings showing a link between HbA1c and elevated predicted whole blood viscosity may have clinical implications. It has been shown that individuals with HbA1c-defined prediabetes exhibit a worse cardiovascular risk profile associated with subclinical organ damage such as vascular stiffness [22], carotid atherosclerosis [6,21], and left ventricular hypertrophy [23], all independent predictors of major cardiovascular events. Increased blood viscosity may represent a unifying mechanism linking elevation in HbA1c concentrations to both type 2 diabetes and cardiovascular disease. Since HbA1c-defined prediabetes has been recognized as a dysglycemic condition requiring careful monitoring with respect to both type 2 diabetes and cardiovascular risk, assessment of subjects employing the HbA1c criterion may be helpful to capture at risk individuals who may benefit from an intensive intervention based on diet, exercise, and/or pharmacotherapy in order to prevent or delay adverse clinical outcomes.

\section{Acknowledgments}

M.A.M., T.V.F.: researched and analyzed data, reviewed and edited the manuscript; F.A., E.S., A.S.: contributed to research data; G.C.M., F.P.: contributed to the discussion and reviewed the manuscript; G.S. designed the study, analyzed the data and wrote the manuscript.

All co-authors have no conflicts of interest.

This work was supported, in part, by the Foundation for Diabetes Research of the Italian Diabetes Society, through the award of "Fondazione Diabete Ricerca FO.DI.RI. - MSD" scholarships in 2014 and 2015 to Gaia Chiara Mannino.

\section{References}

[1] American Diabetes Association. Standards of medical care in diabetes - 2017. Diabetes Care 2017;40(Suppl. 1):S11-24.

[2] Ratner R, Goldberg R, Haffner S, Marcovina S, Orchard T, Fowler S, et al., Diabetes Prevention Program Research Group. Impact of intensive lifestyle and metformin therapy on cardiovascular disease risk factors in the diabetes prevention program. Diabetes Care 2005;28:888-94.

[3] Selvin E, Steffes MW, Zhu H, Matsushita K, Wagenknecht L, Pankow J, et al. Glycated hemoglobin, diabetes, and cardiovascular risk in nondiabetic adults. N Engl J Med 2010;362:800-11.

[4] Zhang X, Gregg EW, Williamson DF, Barker LE, Thomas W, Bullard KM, et al. A1C level and future risk of diabetes: a systematic review. Diabetes Care 2010;33:1665-73.

[5] Olson DE, Rhee MK, Herrick K, Ziemer DC, Twombly JG, Phillips LS. Screening for diabetes and pre-diabetes with proposed A1C-based diagnostic criteria. Diabetes Care 2010;33:2184-9.

[6] Marini MA, Succurro E, Castaldo E, Cufone S, Arturi F, Sciacqua A, et al. Cardiometabolic risk profiles and carotid atherosclerosis in individuals with prediabetes identified by fasting glucose, postchallenge glucose, and hemoglobin A1c criteria. Diabetes Care 2012;35:1144-9.

[7] Marini MA, Succurro E, Arturi F, Ruffo MF, Andreozzi F, Sciacqua A et al. Comparison of A1C, fasting and 2-h post-load plasma glucose criteria to diagnose diabetes in Italian Caucasians. Nutr Metab Cardiovasc Dis 2012;22:561-6.

[8] Ciuffetti G, Schillaci G, Lombardini R, Pirro M, Vaudo G, Mannarino E. Prognostic impact of low-shear whole blood viscosity in hypertensive men. Eur J Clin Invest 2005;35:93-8.

[9] Sloop GD, Garber DW. The effects of low-density lipoprotein and high-density lipoprotein on blood viscosity correlate with their association with risk of atherosclerosis in humans. Clin Sci 1997; 92:473-9.

[10] Ernst E. Haemorheological consequences of chronic cigarette smoking. J Cardiovasc Risk 1995;2:435-9.

[11] Letcher RL, Chien S, Pickering TG, Laragh JH. Elevated blood viscosity in patients with borderline essential hypertension. Hypertension 1983;5:757-62.

[12] Irace C, Carallo C, Scavelli F, De Franceschi MS, Esposito T, Gnasso A. Blood viscosity in subjects with normoglycemia and prediabetes. Diabetes Care 2014;37:488-92.

[13] Høieggen A, Fossum E, Moan A, Enger E, Kjeldsen SE. Whole-blood viscosity and the insulin-resistance syndrome. J Hypertens 1998; 16:203-10.

[14] Wannamethee SG, Perry IJ, Shaper AG. Hematocrit and risk of NIDDM. Diabetes 1996;45:576-9.

[15] Tamariz LJ, Young JH, Pankow JS, Yeh HC, Schmidt MI, Astor B, et al. Blood viscosity and hematocrit as risk factors for type 2 diabetes mellitus: the atherosclerosis risk in communities (ARIC) study. Am J Epidemiol 2008;168:1153-60.

[16] O'Leary DH, Polak JF, Kronmal RA, Manolio TA, Burke GL, Wolfson Jr SK. Carotid artery intima and media thickness as a risk factor for myocardial infarction and stroke in older adults. N Engl J Med 1999;340:14-22.

[17] Fiorentino TV, Sesti F, Andreozzi F, Pedace E, Succurro E, Sciacqua A, et al. Frequency of 1 -h post-load glucose $\geq 155 \mathrm{mg} / \mathrm{dl}$ among individuals with different glucose tolerance conditions. Nutr Metab Cardiovasc Dis 2016;26:439-41.

[18] de Simone G, Devereux RB, Chien S, Alderman MH, Atlas SA, Laragh JH. Relation of blood viscosity to demographic and physiologic variables and to cardiovascular risk factors in apparently normal adults. Circulation 1990;81:107-17.

[19] Minato S, Takenouchi A, Uchida J, Tsuboi A, Kurata M, Fukuo K, et al. Association of whole blood viscosity with metabolic syndrome in type 2 diabetic patients: independent association with post-breakfast triglyceridemia. J Clin Med Res 2017;9:332-8.

[20] Matsuda M, De Fronzo RA. Insulin sensitivity indices obtained from oral glucose tolerance testing: comparison with the euglycemic insulin clamp. Diabetes Care 1999;22:1462-70.

[21] Fiorentino TV, Sesti F, Andreozzi F, Pedace E, Sciacqua A, Hribal ML, et al. One-hour post-load hyperglycemia combined with HbA1c identifies pre-diabetic individuals with a higher cardio-metabolic risk burden. Atherosclerosis 2016;253:61-9.

[22] Di Pino A, Scicali R, Calanna S, Urbano F, Mantegna C, Rabuazzo AM, et al. Cardiovascular risk profile in subjects with prediabetes and new-onset type 2 diabetes identified by $\mathrm{HbA}(1 \mathrm{c})$ according to American Diabetes Association criteria. Diabetes Care 2014;37:1447-53.

[23] Sesti G, Fiorentino TV, Perticone M, Sciacqua A, Andreozzi F, Hribal ML, et al. Characterization of left ventricular mass in individuals at risk for type 2 diabetes identified by HbA1c levels according to the American Diabetes Association Criteria. Int J Cardiol 2015;179:211-3.

[24] Brun JF, Varlet-Marie E, Raynaud de Mauverger E, Mercier J. Minimal model-derived insulin sensitivity, insulin secretion and glucose tolerance: relationships with blood rheology. Clin Hemorheol Microcirc 2012;51:21-7.

[25] Brun JF, Varlet-Marie E, Raynaud de Mauverger E. Relationships between insulin sensitivity measured with the oral minimal model and blood rheology. Clin Hemorheol Microcirc 2012;51:29-34.

[26] Marini MA, Frontoni S, Succurro E, Arturi F, Sciacqua A, Hribal ML, et al. Insulin sensitivity, and $\beta$-cell function in relation to hemoglobin A1C. Nutr Metab Cardiovasc Dis 2014;24:27-33.

[27] Sciacqua A, Grillo N, Quero M, Sesti G, Perticone F. Asymmetric dimethylarginine plasma levels and endothelial function in newly 
diagnosed type 2 diabetic patients. Int J Mol Sci 2012;13: 13804-15.

[28] Tsuda K, Kinoshita Y, Nishio I, Masuyama Y. The role of insulin in the regulation of membrane fluidity of erythrocytes in essential hypertension - an electron paramagnetic resonance investigation. Am J Hypertens 2000;13:376-82.

[29] Fiorentino TV, Hribal ML, Perticone M, Andreozzi F, Sciacqua A, Perticone F, et al. Unfavorable inflammatory profile in adults at risk of type 2 diabetes identified by hemoglobin A1c levels according to the American Diabetes Association criteria. Acta Diabetol 2015;52:349-56.

[30] Herman WH, Ma Y, Uwaifo G, Haffner S, Kahn SE, Horton ES, et al., Diabetes Prevention Program Research Group. Differences in A1C by race and ethnicity among patients with impaired glucose tolerance in the Diabetes Prevention Program. Diabetes Care 2007;30:2453-7. 